\title{
A New Approach to Partial Discharge Detection Under DC Voltage
}

\author{
Pietro Romano, Giuseppe Presti, Antonino Imburgia \\ L.E.PR.E. HV Laboratory, D.E.I.M. Department, \\ Palermo University, \\ Palermo, Italy \\ Roberto Candela \\ Prysmian Electronics Srl \\ Palermo, Italy
}

\begin{abstract}
SUMMARY
A new method for PD detection under HVDC is proposed, based on the application of a periodic continuous waveform. It is suitable for testing HVDC cable systems.
\end{abstract}

\author{
Index Terms - HVDC, partial discharge, DC measurements, testing method, \\ pattern recognition, discharge phenomena
}

\section{INTRODUCTION}

The continuing development of HVDC power transmission systems presents many problems related to evaluation of the reliability of power system assets [1] - [5]. In this context the identification of insulation defects plays a key role in preventing unexpected failures of electrical components. Partial discharge (PD) measurement is a useful approach to assessing the condition of $\mathrm{HV}$ power apparatus and cables. Such measurements are also widely employed for HVAC systems. The inception mechanisms of PD in AC systems are well-known, and measurements are usually performed following the IEC 60270 standard [6].

PD measurements under DC voltage present complexities related to the nature of the phenomenon and the supply conditions [7]. In AC systems phase-resolved-partial-discharge (PRPD) patterns allow assessment of the insulation condition, and provide information on the types of defect present [8]. Such analysis cannot be performed under DC voltage, since each discharge event cannot be related to a phase value. The interpretation of the acquired data therefore requires a different approach.

Fromm was the first researcher to propose an approach for the classification of PD under DC voltage [9]. Other authors [10]-[12] have proposed statistical approaches to the recognition of phenomena generated by artificial defects (internal, surface and corona specimens), using parameters such as the discharge magnitudes $(\mathrm{Q})$ and the time between discharges (TBD). The aim was to extract Weibull distribution parameters fitting the empirical $\mathrm{Q}$ and TBD distributions and use these distributions for defect identification purposes. New pattern recognition methodologies for PD observed under DC stress have been proposed more recently [13], [14].
PRPD patterns acquired under AC conditions allow the investigator to distinguish easily between noise and PD signals. Under DC voltages it is necessary to implement a noise rejection strategy in order to remove noise signals from acquired data. Niu et al [15] proposed a strategy based on cross- correlation analysis.

The $\mathrm{PD}$ repetition rate under $\mathrm{AC}$ is orders of magnitude higher than that under DC with the same amplitude [9]. For this reason, some DC tests have been performed applying positive and negative voltage ramps [16].

In order to acquire enough data for diagnosis under DC, voltages higher than the corresponding $\mathrm{AC}$ voltages need to be applied, and for longer times [7]. In a recent paper [17] Montanari et al suggested that adoption of time-frequency (TF) maps for PD phenomena identification, and separation algorithms developed for AC PD measurements, might prove effective for DC work.

In this paper we propose a new approach to PD measurement under DC voltage. It offers the possibility of building PRPD-like patterns, using analysis similar to that adopted for AC, and overcomes some of the problems mentioned above. No standard for HVDC PD tests exists at present.

\section{THE VOLTAGE WAVEFORM}

To overcome the problem of PD synchronization with the DC voltage, a new DC waveform has been introduced. It has a positive or negative average value (Figure 1), and is derived from a single-phase half-wave rectifier with added variable offset $\delta \mathrm{V}_{\mathrm{x}}$. It is defined mathematically by

$v(t)=\left\{\begin{array}{c}V_{x} \sin (\omega t) \quad \text { for } \alpha \leq \omega t \leq \pi-\alpha \\ \delta V_{x} \quad \text { for } 0 \leq \omega t<\alpha, \quad \pi-\alpha<\omega t \leq 2 \pi\end{array}\right.$ 
where $\delta V_{x}$ takes values between 0 and $V x$. It follows a sinusoidal law from $\alpha$ to $\pi-\alpha$ and is constant over the remainder of the period.

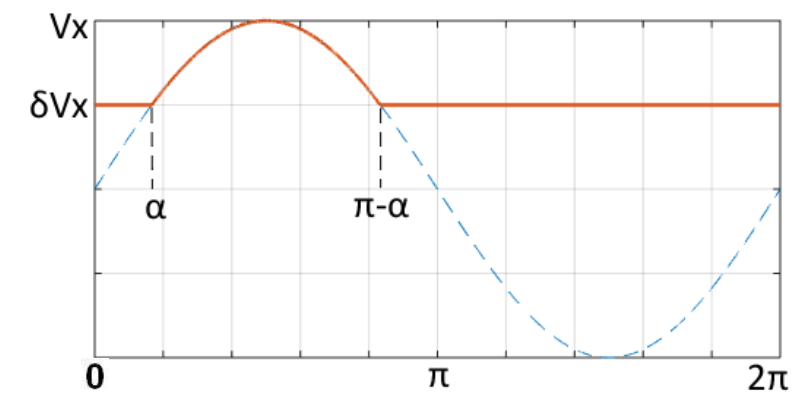

Figure 1: Voltage waveform proposed for PD tests under DC voltage.

The average and rms values of the voltage $\left(\mathrm{V}_{\mathrm{a}}\right.$ and $\mathrm{V}_{\mathrm{rms}}$ respectively) can be varied by varying the parameter $\delta$ over the range $0-1$. We have

$$
\begin{aligned}
& V_{a}=\frac{V_{x}}{2 \pi}[\delta(2 \alpha+\pi)+2 \cos (\alpha)] \\
& V_{r m s}=V_{x} \sqrt{\frac{1}{2 \pi}\left\{\delta^{2}(2 \alpha+\pi)+\frac{1}{2}[\pi-2 \alpha+\sin (2 \alpha)]\right\}}
\end{aligned}
$$

where $\alpha=\arcsin (\delta)$. Given $\delta=0.625, \mathrm{~V}_{\mathrm{rms}}=0.707 \mathrm{~V}_{\mathrm{x}}$, corresponding to the RMS value of a sinusoidal voltage with the same peak value (Figure 2). Figure 3 shows $V_{\text {rms }}$ versus $\mathrm{V}_{\mathrm{x}}$ for various values of $\delta ; \delta=1$ coincides with the DC case and $\delta=0.625$ coincides with the AC case. The latter statement is the well-known equivalence relationship between RMS value and DC value, and does not imply any other similarity between the AC and DC cases.

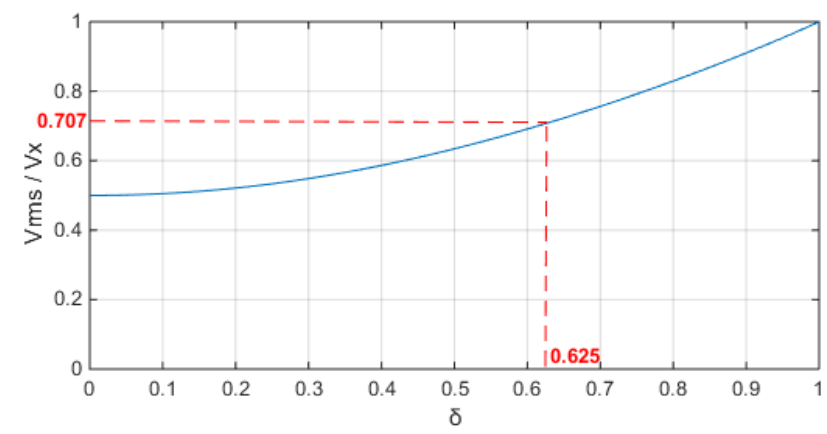

Figure 2: $\mathrm{V}_{\mathrm{rms}} / \mathrm{V}_{\mathrm{x}}$ versus $\delta$

Using this periodic waveform it is possible to acquire a 50 $\mathrm{Hz}$ synchronization signal from which a PRPD pattern can be plotted. Pattern recognition criteria similar to those adopted for AC PD phenomena can then be developed. Frequency can also be used as an additional parameter for varying the test conditions.

The aim in adopting this waveform was to have a unipolar electric field distribution, so that electrons available for the partial discharge process are generated by the space charge distribution inside the material, and in the interface of the material with the cavity. In this way an electric field distribution and electrons are generated, as in DC.

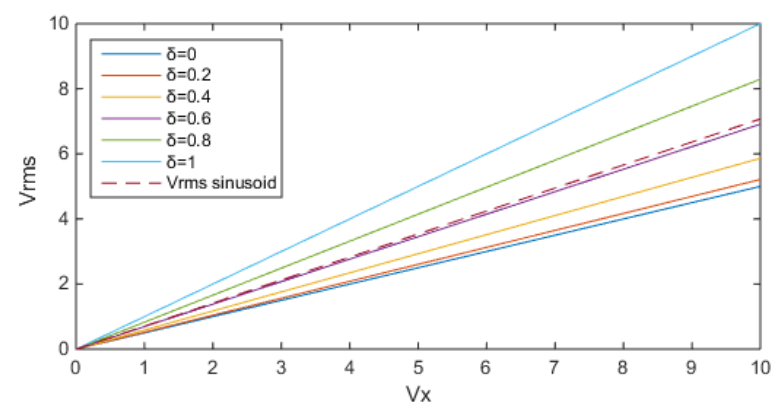

Figure 3: $V_{r m s}$ versus $V_{x}$ for different values of $\delta$.

Recommendations for testing HVDC extruded cable systems will be found in Cigré TB 496 [18]. Standards (not specific for HVDC) have also been issued by IEC [19], and recommendations by Electra [20].

In many tests, the extruded cable system (cable, joints and terminations) form a closed loop in order to ensure that the tests are performed under real load conditions, with current generated using specific transformers. The temperature drop across the insulation is reproduced not by applying a voltage source, but by supplying current. In this way PD measurements can be made taking into account both temperature variation and space charge accumulation under realistic operating conditions.

An HVDC periodic source could be made using the circuit shown in Figure 4. It consists of a high voltage transformer and a single-phase full-bridge rectifier, the output of which is regulated by a control circuit which drives two insulated-gate bipolar-transistors (IGBTs) $\mathrm{S}_{1}$ and $\mathrm{S}_{2}$ (or more depending on the required voltage level). With $S_{1}$ on and $S_{2}$ off, the

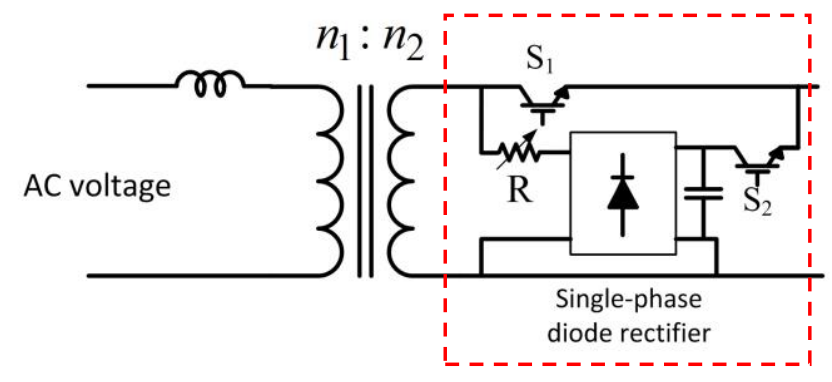

Figure 4: HVDC periodic source.

sinusoidal waveform shown in the Figure 1 in the interval $\alpha$ $-(\pi-\alpha)$ is generated. With $S_{1}$ off and $S_{2}$ on, the single phase full bridge rectifier is connected to the output through a large capacitor filter and generates a pure DC voltage at the output, as shown in Figure 1 in the intervals $0-\alpha$ and $(\pi-\alpha)-2 \pi$.

\section{EXPERIMENTAL SETUP}

The measurement set-up used for the experimental tests is shown in Figure 5. The test cells adopted in the present work were such that internal, surface and corona discharges could be initiated within them (Figure 6). The internal discharge test cell consisted of two $127 \mu \mathrm{m}$ thick overlapping Kapton layers, one of which had been drilled in order to simulate a cavity. The cell was immersed in a container filled with silicone oil, in order to avoid inception of surface discharges over the area of the Kapton layers protruding 
over the electrodes (Figure 6 (a), (b)). A similar test cell was used to simulate surface discharges, but only one Kapton layer was interposed between the two electrodes with different diameters (Figure 6 (c)). Corona discharges were obtained using the classic air-insulated point-plane gap geometry (Figure $6(\mathrm{~d})$ ).

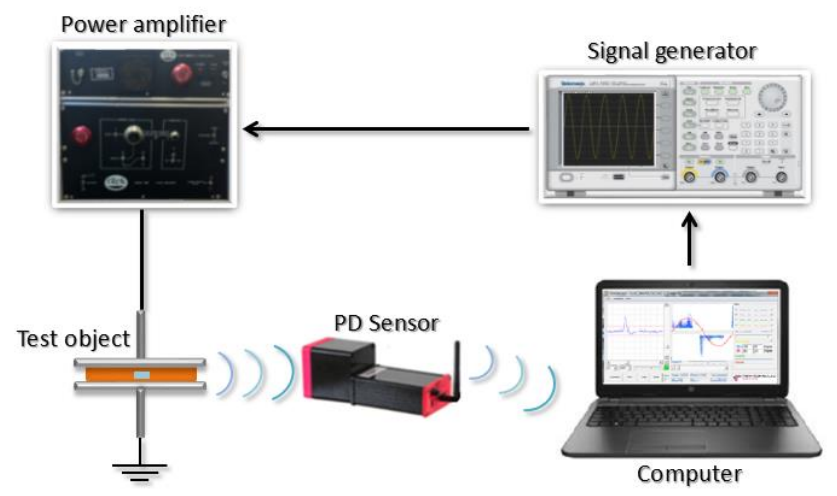

Figure 5: Measurement set-up.

In order to investigate the ability of the set-up to recognize multiple and concurrent phenomena, some measurements were performed with two cells connected together (see below).
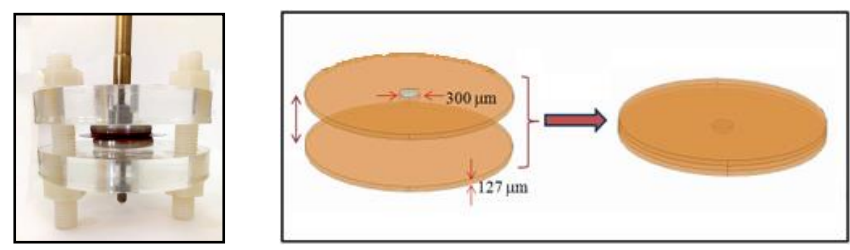

a) Internal discharge specimen with two overlapping Kapton layers.

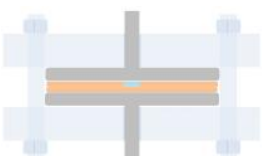

b) Internal specimen

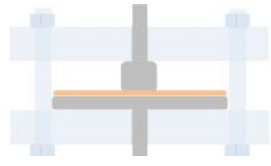

c) Surface specimen

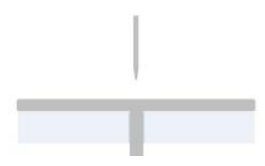

d) corona specimen
Figure 6: Test cells used to simulate partial discharge phenomena.

The continuous voltage waveform was generated by a Tektronix signal generator, with maximum output signal amplitude $\pm 10 \mathrm{~V}$, connected to a Trek Model 664 power amplifier capable of amplifying the input signal by 1000 . PD signals were acquired using a wireless sensor Prysmian Pry-Cam, which has a bandwidth of $100 \mathrm{MHz}$ and transmits the acquired data to the software PDiscover. The latter uses a WiFi communication protocol [21], [22]. The sensor was placed at a distance of $10 \mathrm{~cm}$ from the cable connecting the test cell to ground. The Trek 664 power amplifier could also function as a constant DC voltage source, and was used to l compare the results obtained using the proposed waveform with those obtained using a constant voltage.

Unfortunately the maximum applied voltage available was insufficient to enable us to study other materials and other types of defects.

\section{EXPERIMENTAL RESULTS}

\subsection{Test procedure}

In order to verify that the proposed method yields reliable results, we compared the results obtained by (a) stressing the internal artificial void specimen with a constant DC voltage, (b) applying a sinusoidal voltage, and (c) applying the waveform shown in Figure 1. The inception voltage and the discharge repetition rate were recorded for each test. More specifically, the variation of these two quantities with $\delta$ was recorded. When $\delta$ is increased from 0 to 1 , the ripple on the DC voltage decreases and the voltage is almost constant. PRPD patterns were also acquired and analyzed using the software PDiscover. Finally, internal - corona and internal - surface cells were combined in order to verify that patterns acquired using the Figure 1 waveform can be used to identify various PD phenomena, thereby simplifying the statistical analysis of the data.

\subsection{AC results}

The AC tests were performed using a $50 \mathrm{~Hz}$ sinusoidal waveform with an rms value of $5 \mathrm{kV}$. The resulting PRPD pattern for an internal discharge sample (Figure 7) shows the expected behavior, with "rabbit-like" shape typical of internal discharges. The discontinuities in discharge magnitude after both voltage polarity inversions (transition from "rabbit-ear" to "rabbit-body") are mainly due to reduced electron availability, leading to a delay in PD inception (PDIV) [23]. The PD repetition rate recorded during the measurements was about 66 pulses/sec, and the inception voltage $V_{x}$ was about $2 \mathrm{kV}$. The vertical color scale, showing the number of discharges with the same magnitude and phase, runs from 1 to 250 discharges in steps of 3.5 discharges.

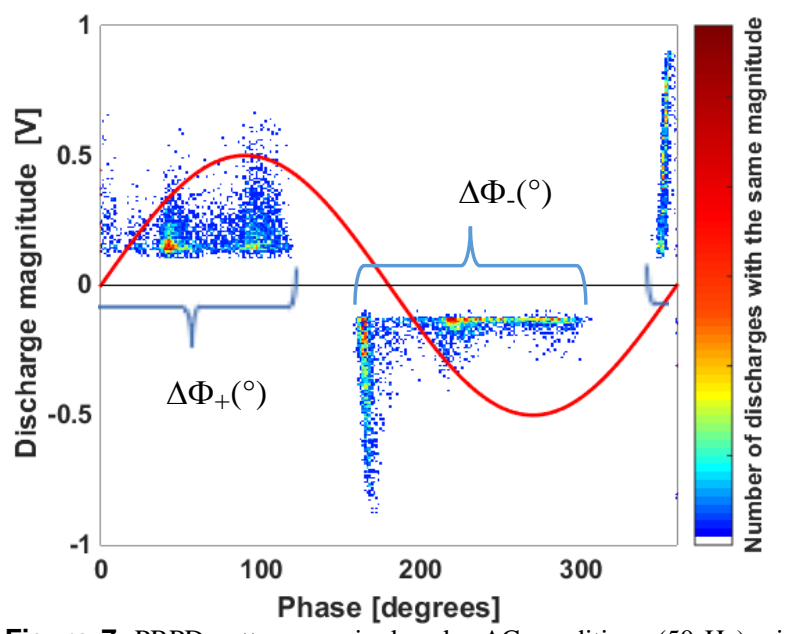

Figure 7: PRPD pattern acquired under AC conditions $(50 \mathrm{~Hz})$ with an applied voltage $V_{r m s}=5 \mathrm{kV}\left(V_{x}=7.07 \mathrm{kV}\right)$ on an artificial internal discharge specimen. The red line shows the applied voltage, while $\Delta \Phi_{+}$ and $\Delta \Phi_{-}$show the width of the spectrum for positive and negative PDs respectively.

\subsection{Constant DC results}

The DC test was performed with constant voltages of 5 $\mathrm{kV}$ and $10 \mathrm{kV}$ (Trek 884), and no PD activity was detected. The test was repeated with a Fug HCN-14 DC voltage generator $(20 \mathrm{kV})$; the PDIV was $14 \mathrm{kV}$, and the PD pattern observed at this voltage is shown in Figure 8. The total 
number of acquired discharges was 101 ,

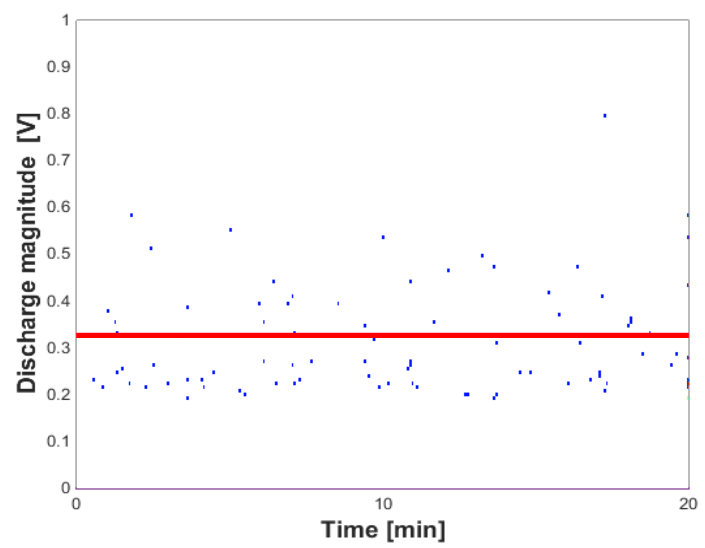

Figure 8: PD pattern obtained under $14 \mathrm{kV}$ DC voltage on an artificial internal discharge sample. The continuous red line shows the DC applied voltage.

corresponding to a repetition rate of $0.084 \mathrm{pulses} / \mathrm{sec}$.

\subsection{Periodic waveform results}

The direct current periodic (DCP) tests using the continuous waveform were performed with $\delta=0.625$. With this waveform, repetitive PDs were first detected at $V_{x}=5.8 \mathrm{kV}$ (PDIV), following which PD acquisition was performed at | $V_{x}=7.07 \mathrm{kV}$, in order to obtain an $r m s$ value of $5 \mathrm{kV}$. Figure 9 shows the associated PRPD pattern, the vertical color scale being the same as that in Figure 7 . The acquisition time was about $60 \mathrm{sec}$. Under these conditions it was possible to acquire partial discharges synchronized with the DCP waveform, as in the AC case (Figure 7), while the discharges in the DC case (Figure 8) were acquired over a 20 minute period.

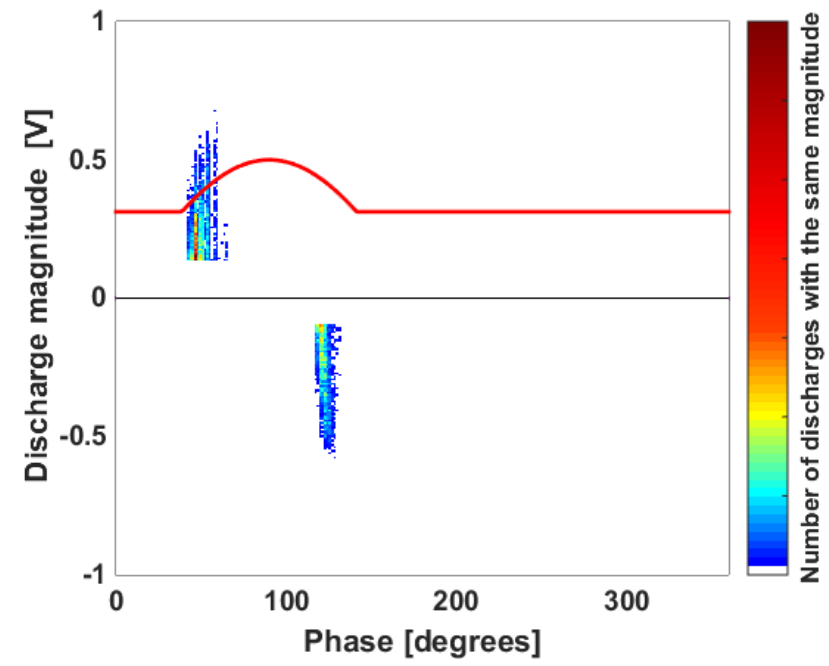

Figure 9: PD pattern acquired using the DCP waveform $(\mathrm{f}=50 \mathrm{~Hz}$, $\delta=0.625, V_{r m s}=5 \mathrm{kV}, V_{x}=7.07 \mathrm{kV}$ ) and an artificial internal discharge specimen. The acquisition time was about $60 \mathrm{sec}$. The red line shows the DC periodic applied voltage.

The presence of both negative and positive discharges should be noted. Only one polarity would be expected since the applied voltage is unipolar. It is well know that the polarity of AC PDs is linked to the sign of the voltage derivative $d v / d t$ [24]. Accordingly, since positive and negative $d v / d t$ are present in the DCP waveforms, positive and negative discharges are generated. Application of an electric field greater than that used in the present work might result in unipolar discharges, because of the influence of space charge accumulated on the dielectric/air interface [25].

PD pulses are generated when $d v / d t$ is not zero, and the amplitudes of the acquired discharges are similar to those observed in the AC tests (compare Figures 7 and 9). The absence of a zero crossing in the DCP waveform would be expected to reduce the width of the PD spectrum for both positive and negative discharges. In particular, it is expected that, for positive discharges, the DCP waveform (with no positive $d v / d t$ values when the voltage is negative) will cause the disappearance of the "ear" part of the pattern, and for negative discharges, the DCP waveform (with no negative $d v / d t$ values when the voltage is negative) will cause the disappearance of the "body" part of the pattern. This reduction occurs mainly for internal discharges in which an inception phase angle less than the phase angle of zero crossing can often be observed [23].

Figures 10 and 11 show pulse shapes and admittance amplitude harmonic spectra of the PDs, under the AC and DCP applied voltages respectively. These Figures are very similar, confirming that the artificial internal defect is the same under both waveforms.
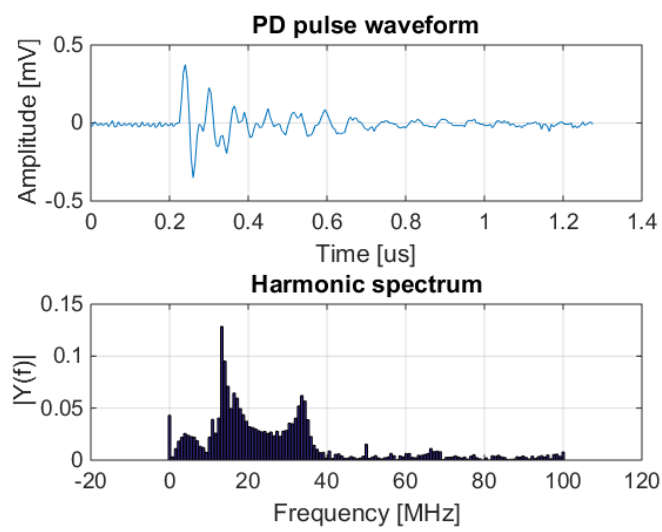

Figure 10: Internal PD pulse shape and admittance amplitude harmonic spectrum observed under the $\mathrm{AC}$ waveform.
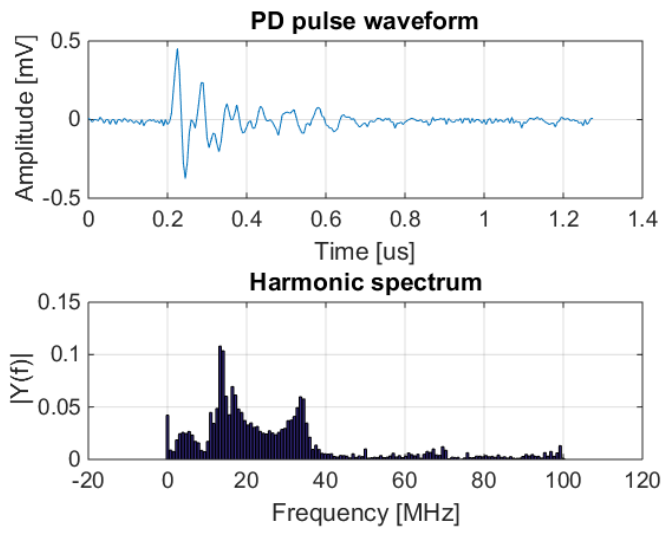

Figure 11: Internal PD pulse shape and admittance amplitude harmonic spectrum observed under the DCP waveform. 


\subsection{Dependence of PDIV and $P D$ repetition rate on $\delta$}

As explained in Section 2, the parameter $\delta$ links the AC case $(\delta=0.625)$ and the DC case $(\delta=1)$. The dependence of the PDIV and repetition rate on $\delta$ was investigated at $V_{x}=7.07 \mathrm{kV} .10000$ pulses were acquired for each value of $\delta$. The results are presented in Table 1. PDIV and PD repetition rate (PDRR) variation with $\delta$ are shown in Figures 12 and 13 respectively.

The inception voltage increases steadily when $\delta$ increases from zero to 0.8 , i.e., as the sinusoidal part of the waveform varies between $\alpha$ and $\pi-\alpha$ (Figure 1). The width of positive and negative discharge clusters and the discharge maximum magnitudes decrease as the voltage waveform tends to a constant $(\delta \rightarrow 1)$. The discharge repetition rate decreases steadily as $\delta$ increases. No PDs were detected for $\delta=0.9$ and $\delta$ $=1$. It may be that the inception voltages at these $\delta$ values are higher than $10 \mathrm{kV}$.

Table I: PD inception voltage PDIV and discharge repetition rates PDRR for various values of $\delta$, and repetition rates under AC and DC (Sections 4.2 and 4.3$)$.

\begin{tabular}{|c|c|c|c|c|}
\hline$\delta$ & $\begin{array}{c}\text { PDIV } \\
{[\mathrm{kV}]}\end{array}$ & $\begin{array}{c}\mathrm{PD} \\
\text { repetition } \\
\text { rate under } \\
\mathrm{DCP} \\
{[\mathrm{PD} / \mathrm{s}]}\end{array}$ & $\begin{array}{c}\mathrm{PD} \\
\text { repetition } \\
\text { rate under } \\
\mathrm{AC}(5 \mathrm{kV}) \\
{[\mathrm{PD} / \mathrm{s}]}\end{array}$ & $\begin{array}{c}\text { PD repetition } \\
\text { rate under DC } \\
(14 \mathrm{kV}) \\
{[\mathrm{PD} / \mathrm{s}]}\end{array}$ \\
\hline 0 & 2.2 & 55.37 & \multirow{11}{*}{66.40} & \multirow{11}{*}{0.084} \\
\hline 0.1 & 2.6 & 47.34 & & \\
\hline 0.2 & 2.9 & 37.87 & & \\
\hline 0.3 & 3.4 & 30.92 & & \\
\hline 0.4 & 3.7 & 23.40 & & \\
\hline 0.5 & 4.4 & 18.35 & & \\
\hline 0.6 & 5.7 & 12.34 & & \\
\hline 0.7 & 7.5 & 9.55 & & \\
\hline 0.8 & 9.8 & 0.45 & & \\
\hline 0.9 & - & - & & \\
\hline 1 & - & - & & \\
\hline
\end{tabular}

Figure 12 and 13 also show the results of fitting second order polynomials in $\delta$ to the experimental PDIV and PD repetition rate data respectively. The polynomials were

$\mathrm{PVID}=14.15 \delta^{2}-2.71 \delta+2.56$

$\mathrm{PDRR}=27.48 \delta^{2}-88.10 \delta+55.19$

The correlation coefficients $\mathrm{R}^{2}$ were 0.9941 and 0.9954 for (4) and (5) respectively. As far as we are aware, there is no widely accepted theoretical reason why the PDIV and PDRR should follow a second order polynomial in $\delta$. We chose it as the simplest mathematical expression yielding a good fit to the experimental data. However, first order polynomials in $\delta$ also yielded good fits to the experimental data with $\mathrm{R}^{2}$ values of 0.8688 and 0.9866 for PDIV and PDRR respectively.

Figure 12 suggests that the PDIV corresponding to a constant DC voltage is approximately $14 \mathrm{kV}$ at $\delta=1$, in agreement with the PDIV evaluated in the constant DC test (Section 4.3).
Figure 13 shows that the PDRR decreases at a nearly constant rate from $\delta=0$ to $\delta=0.8$. Substitution of $\delta=1$ (constant DC, Section 4.3) in (5) yields PDRR $\approx-5 \mathrm{PD} / \mathrm{s}$, qualitatively consistent with the very small experimental rate of $0.084 \mathrm{PD} / \mathrm{s}$.

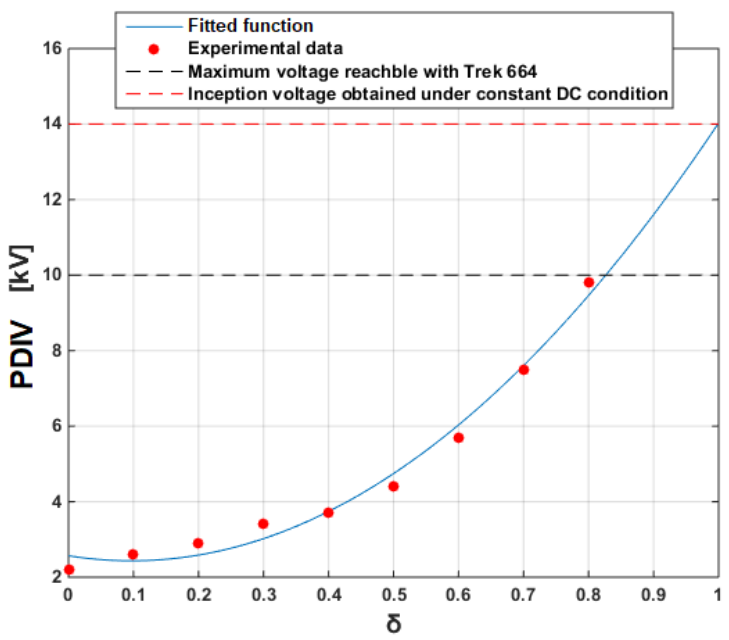

Figure 12: Variation of measured PDIV with $\delta$ (red circles), and a second order polynomial in $\delta$ fitted to the experimental data (solid blue curve).

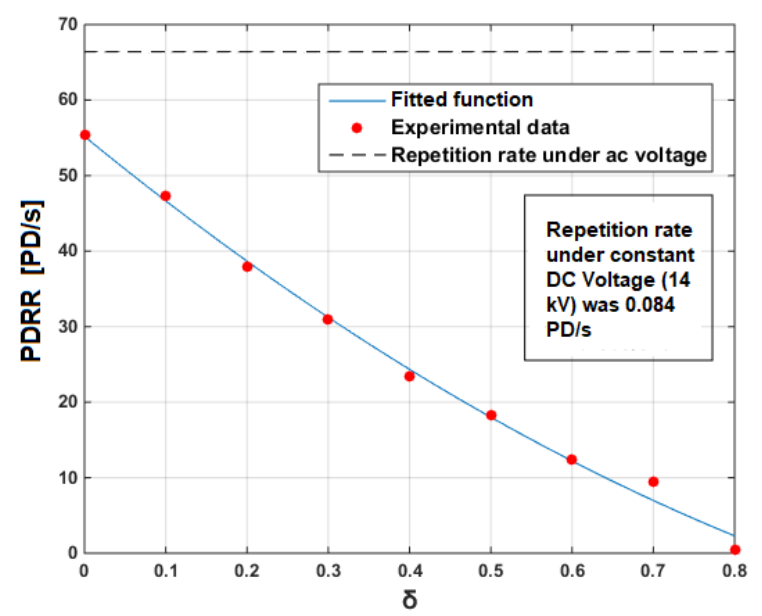

Figure 13: Variation of measured PD repetition rate (PDRR) with $\delta$ (red circles), and a second order polynomial in $\delta$ fitted to the experimental data (solid blue curve).

Figure 14 shows the PD patterns obtained using the internal discharge specimen, over the range $\delta=0$ to $\delta=0.8$. The phase angle at which positive discharges commence increases from about $10^{\circ}$ to about $60^{\circ}$ over this $\delta$ range. No discharges were detected close to the maximum value of the DCP waveform, as often happens with internal defects [26]. Negative discharges appear around $130^{\circ}$ for all values of $\delta$. The negative discharge extinction phase angle lies in the range $127-180^{\circ}$ for all $\delta$ values. The decreasing width of both positive and negative discharge phase angle ranges is closely correlated with the decreasing width of the $\alpha-$ $(\pi-\alpha)$ part of the DCP waveform. At $\delta=0.1$ negative discharges occur after the input voltage has decreased to a constant value, similar to the behavior ("echo PD") reported in [27]. The occurrence of PDs at much the same phase angles for all values of $\delta$ strongly suggests that they are generated by 
the same phenomena, and are not the result of random noise in the apparatus.
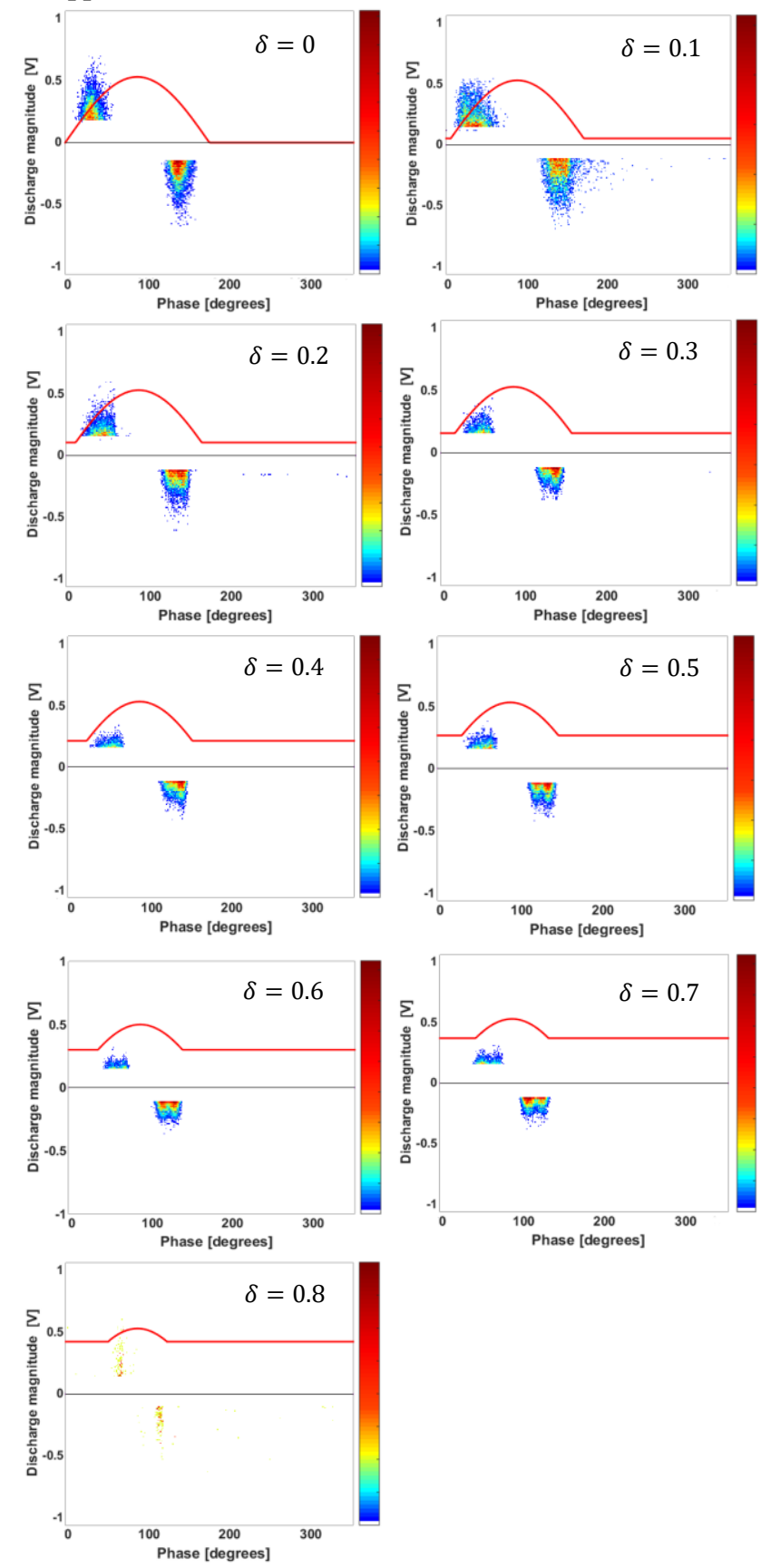

Figure 14: PD patterns acquired for nine values of $\delta$, using the internal discharges cell.

A model which we proposed some years ago [28], taking into account the effect of temperature on the dynamics of PDs in a cavity within a dielectric, may be consistent with the present results [29]. Using the PEA space charge measurement technique we found that the space charge profiles in a sample consisting of a single kapton layer, following application of DCP $(\delta=0.6)$ and DC waveforms, were very similar, assuming that both waveforms have equivalent effect in terms of charge distribution, determining the same electric field gradient. Consequently. in the presence of a void embedded within the dielectric subjected to DCP, the charge, which will also appear at the dielectric/air interface, can contribute to the electron availability and the localized field enhancement, leading to PDs [30].

In this article, PDIVs, PD repetition rates and PD patterns were obtained only for internal discharge specimens.

\subsection{Detection of combined PD phenomena}

PD measurements on real components often reveal the simultaneous presence of various PD phenomena. Correct diagnosis of a dangerous insulation defect requires a reliable PD pattern recognition strategy. The measurement circuit must be designed to avoid inducing corona or surface partial discharges. As discussed in the Introduction, PD data analysis under constant DC voltage requires statistical strategies or techniques [31]-[33] based on wavelet transformation [34]. The method proposed in the present work facilitates defect recognition using PRPD pattern analysis and fast Fourier transforms of PD pulses.

We now present the results of two sets of experimental measurements, the first set performed on a specimen in which corona and internal discharges were occurring simultaneously, and the second set on two specimens connected in parallel in order to simulate simultaneous internal and surface PDs.

Figure 15 shows the pattern acquired in the first set, with $\delta=0.3$ at $8.3 \mathrm{kV}$. One of the three pink rectangles is associated with corona discharges, and the other two with internal discharges. As observed under sinusoidal voltages

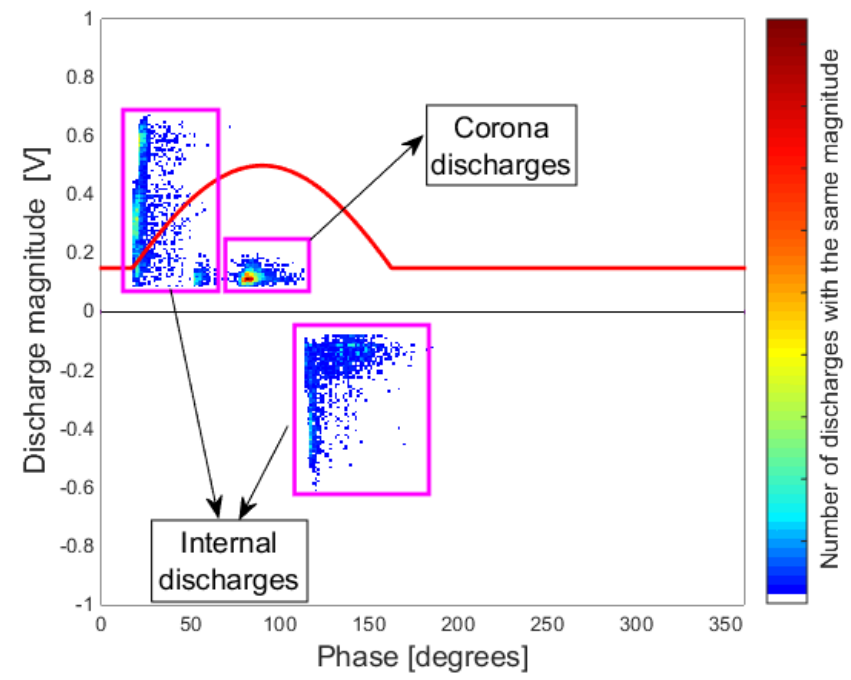

Figure 15: Combined PD patterns acquired by applying the DC periodic waveform $\left(f=50 \mathrm{~Hz}, V_{r m s}=8.3 \mathrm{kV}, \delta=0.3\right)$ to a specimen containing corona and internal defects.

[8],[35], corona discharges are clustered around the maximum value of the applied voltage, while internal discharges are clustered around the region of maximum $d v / d t$ of the applied voltage, as shown in Sections 4.4 and 4.5.

The results shown in Figure 15 can be plotted as a timeresolved PD (TRPD) pattern, in order to clarify how the present approach can simplify the recognition of superimposed PD phenomena. Figure 16 shows that corona and internal discharges are mixed along the time line and cannot be separated using the PD time pattern. 


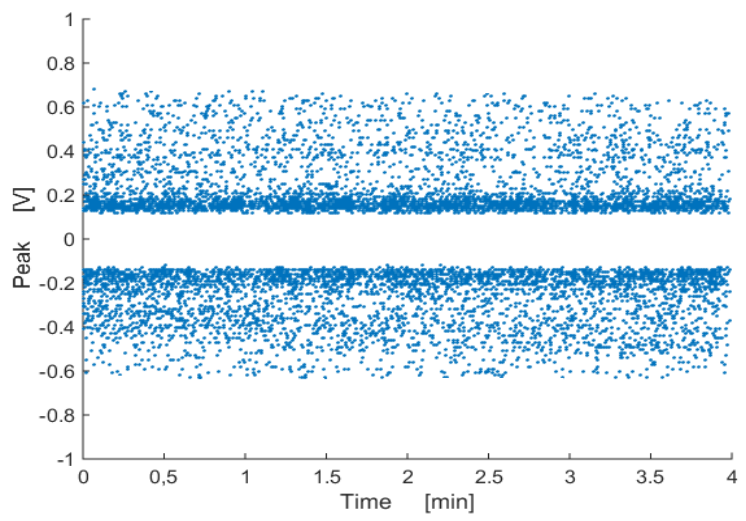

Figure 16: TRPD pattern of combined corona and internal discharges.

The pulse shapes and the corresponding harmonic spectra are compared in Figure 17. The amplitudes of the corona discharges ( $a$ and $b$ ) in the range $0-40 \mathrm{MHz}$ are mostly lower than those of the internal discharges (c and d), consistent with the results of other authors [36]. The peaks around $100 \mathrm{MHz}$ (b and d) are thought to be due to background noise.

Figure 18 shows the PD patterns observed when two specimens were connected in parallel in order to simulate simultaneous internal and surface PDs. It is possible to distinguish between surface and internal PD sources within the four pink rectangles. As in Figure 15, the internal discharges tend to be clustered around the regions of maximum $d v / d t$ in the applied voltage. The surface discharges cluster around one of? the regions of maximum $d v / d t$ in the applied voltage for positive discharges, but for negative discharges they occur more frequently in the constant part of the DCP waveform.

The pulse shapes and the corresponding harmonic spectra, for surface and internal PDs, are presented in Figure 19. The surface PD pulse shown in Figure 19a has a maximum admittance amplitude value around $10 \mathrm{MHz}$ (Figure 19b), while the internal discharge pulse shown in Figure 19c has a maximum admittance amplitude value around $20 \mathrm{MHz}$ (Figure $19 \mathrm{~d}$ ). Results in agreement with this were obtained in measurements made using the same antenna sensor as in the present work, showing a lower maximum admittance amplitude value for surface PDs than for internal PDs [37].
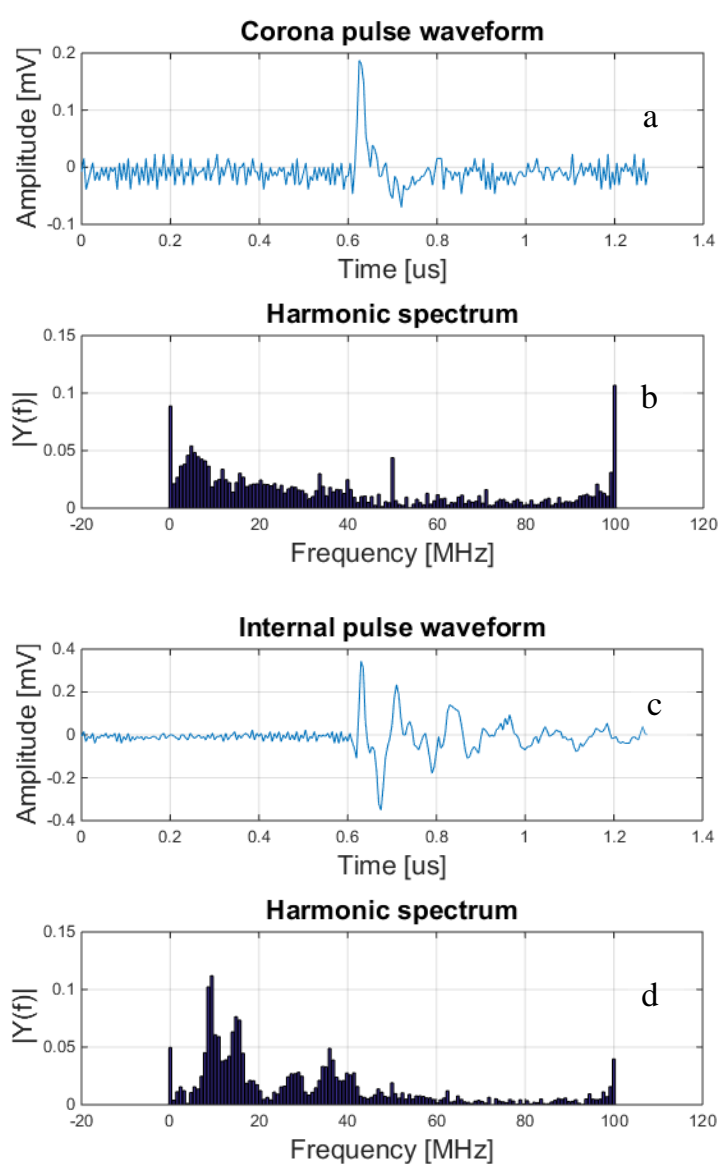

Figure 17: Pulse waveforms and admittance amplitude harmonic spectra for simultaneous corona discharges ( $\mathrm{a}$ and $\mathrm{b}$ ) and internal discharges ( $\mathrm{c}$ and d).

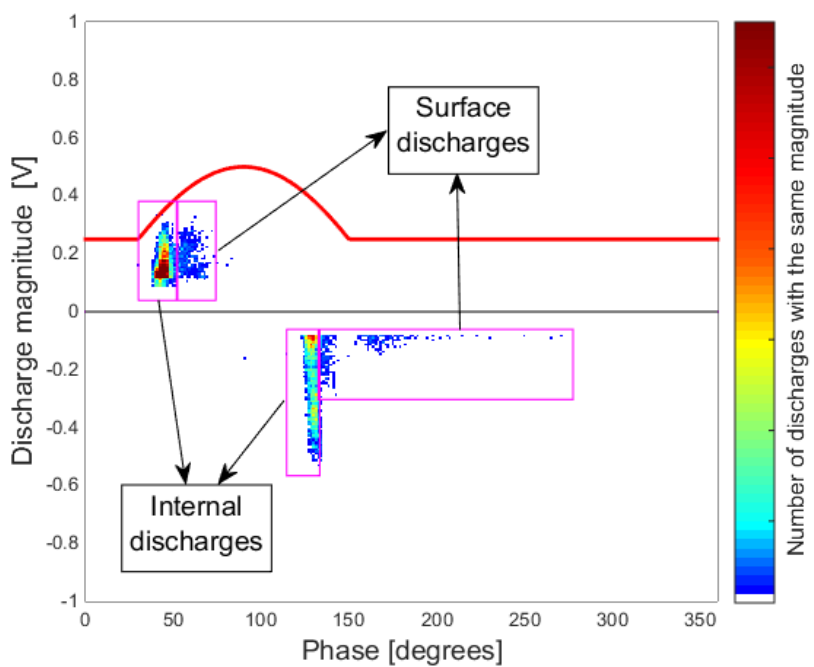

Figure 18: Combined surface and internal discharge PD patterns acquired by applying the DC periodic waveform ( $\mathrm{f}=50 \mathrm{~Hz}, V_{r m s}=7 \mathrm{kV}, \delta=0.5$ ) to a specimen containing surface and internal defects. 

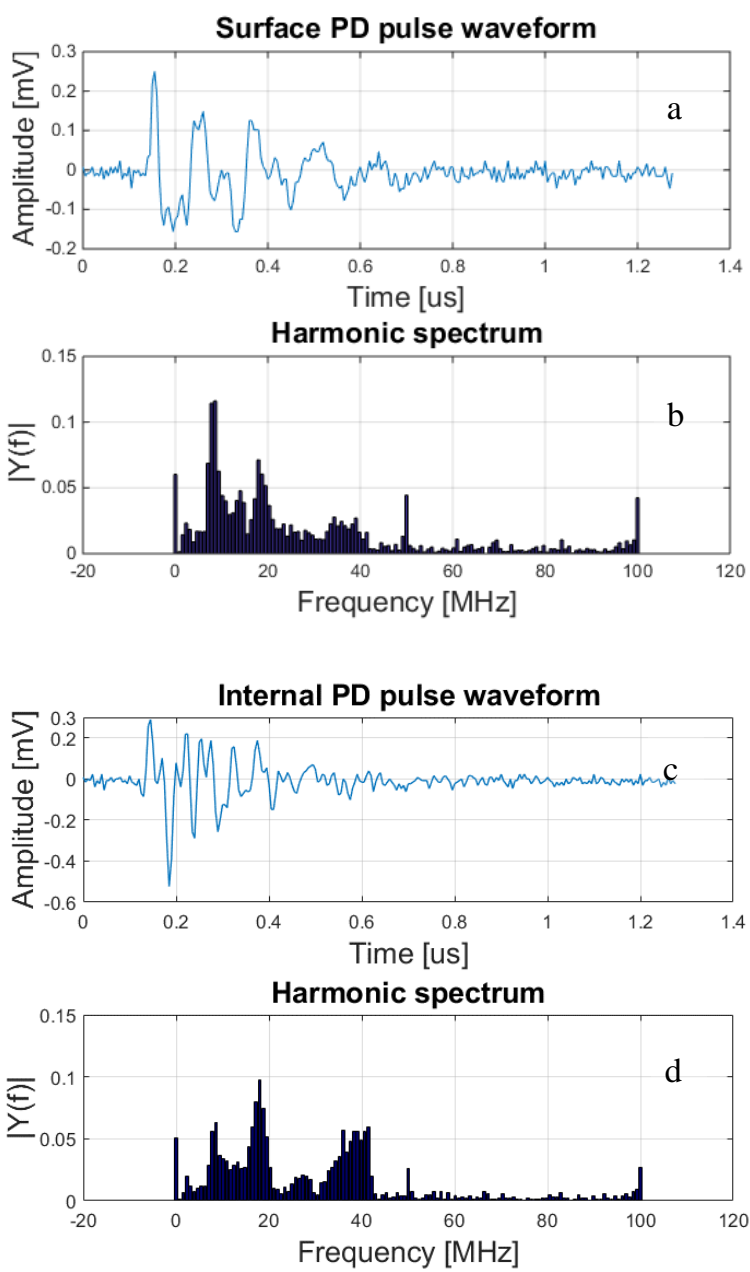

Figure 19: Pulse waveforms and admittance amplitude harmonic spectra for simultaneous surface discharges $(\mathrm{a}$ and $\mathrm{b})$ and internal discharges $(\mathrm{c}$ and d), observed on two specimens connected in parallel.

\section{CONCLUSION}

In this article a new test procedure for partial discharge detection under DC voltage has been proposed. The experimental results show that the direct current periodic waveform overcomes some of the measurement problems associated with "pure" DC. Consequently we suggest that this procedure could be a suitable starting point for the development of PD measurements under DC conditions.

Further work is certainly needed, e.g., measurement of PDIVs and repetition rates in other dielectric materials, and comparison of space charge profiles obtained under classic HVDC waveforms and under the continuous periodic DC waveforms proposed in this work. In addition a test setup operating over a range of frequencies, and capable of applying continuous voltage waveforms with magnitudes up to several hundred $\mathrm{kV}$, needs to be developed.

\section{ACKNOWLEDGMENTS}

The authors would like to thank the Electric Power Engineering Division of the Chalmers University of Technology, Goteborg, Sweden, and in particular Amir Parastar, for their contribution to the design of the converter required to generate the voltage waveform shown in the paper. They also thank Professor Robert Fleming for editorial assistance.

\section{REFERENCES}

[1] U. Astrom, L. Weimers, V. Lescale, and G. Asplund, "Power transmission with HVDC at voltages above 600 $\mathrm{kV}, "$ in 2005 IEEE/PES Transmission \& Distribution Conference \& Exposition:Asia and Pacific, 2005,pp.1-7.

[2] G. Mazzanti; M. Marzinotto, "Improved Design of HVDC Extruded Cable Systems," in Extruded Cables for High-Voltage Direct-Current Transmission: Advances in Research and Development, 1, Wiley-IEEE Press, 2013, pp.384-doi: 10.1002/9781118590423.ch05.

[3] M. Mammeri and B. Dhuicq, "Challenges of extruded cable for HVAC and HVDC power transmission," in PowerTech (POWERTECH), 2013 IEEE Grenoble, 2013, pp. 1-8.

[4] J. C. Fothergill, "The coming of Age of HVDC extruded power cables," in 2014 IEEE Electrical Insulation Conference (EIC), 2014, pp. 124-137.

[5] P. Morshuis, A. Cavallini, D. Fabiani, G. C. Montanari, and C. Azcarraga, "Stress conditions in HVDC equipment and routes to in service failure," IEEE Transactions on Dielectrics and Electrical Insulation, vol. 22, no. 1, pp. 81-91, 2015.

[6] IEC 60270:2000+AMD1:2015 CSV, High-voltage test techniques - Partial discharge measurements.

[7] P. H. Morshuis and J. J. Smit, "Partial discharges at DC voltage: their mechanism, detection and analysis," IEEE Transactions on Dielectrics and Electrical Insulation, vol. 12, no. 2, pp. 328-340, 2005.

[8] E. Gulski, "Digital analysis of partial discharges," in IEEE Transactions on Dielectrics and Electrical Insulation, vol. 2, no. 5, pp. 822-837, Oct 1995. doi: 10.1109/94.469977.

[9] U. Fromm, "Interpretation of partial discharges at DC voltages," IEEE transactions on Dielectrics and Electrical Insulation, vol. 2, no. 5, pp. 761-770, 1995.

[10] H. Niu, A. Cavallini, and G. Montanari, "Identification of partial discharge phenomena in HVDC apparatus," in Electrical Insulation, 2008. ISEI 2008. Conference Record of the 2008 IEEE International Symposium on, 2008, pp. 373-376.

[11] A. Cavallini, G. C. Montanari, M. Tozzi and X. Chen, "Diagnostic of HVDC systems using partial discharges," in IEEE Transactions on Dielectrics and Electrical Insulation, vol. 18, no. 1, pp. 275-284, February 2011.

[12] M. Rahimi, R. Javadinezhad, and M. Vakilian, "DC partial discharge characteristics for corona, surface and void discharges," in 2015 IEEE 11th International Conference on the Properties and Applications of Dielectric Materials (ICPADM), 2015, pp. 260-263.

[13]I. J. Seo, U. A. Khan, J. S. Hwang, J. G. Lee and J. Y. Koo, "Identification of Insulation Defects Based on Chaotic Analysis of Partial Discharge in HVDC Superconducting Cable," in IEEE Transactions on Applied Superconductivity, vol. 25, no. 3, pp. 1-5, June 2015.doi: 10.1109/TASC.2015.2394245. 
[14]A. Pirker and U. Schichler, "Partial discharges at DC voltage - measurement and pattern recognition," 2016 International Conference on Condition Monitoring and Diagnosis (CMD), Xi'an, China, 25-28 Sept. 2016, pp. 287-290, doi: 10.1109/CMD.2016.7757811

[15]H. Niu, A. Cavallini, G. Montanari, and Y. Zhang, "Noise rejection strategy and experimental research on partial discharge at DC voltage," in 2009 IEEE 9th International Conference on the Properties and Applications of Dielectric Materials, 2009, pp. 489492.

[16]E. Corr, W. Siew, and W. Zhao, "PD Activity in Void Type Dielectric Samples for Varied DC Polarity." 2016 IEEE Conference on Electrical Insulation and Dielectric Phenomena (CEIDP), Toronto, ON, 2016, pp. 510-513. doi: 10.1109/CEIDP.2016.7785616

[17] R. Aldrian, G. C. Montanari, A. Cavallini and Suwarno, "Signal separation and identification of partial discharge in XLPE insulation under DC voltage," 2017 1st International Conference on Electrical Materials and Power Equipment (ICEMPE), Xi'an, 2017, pp. 5356. doi: 10.1109/ICEMPE.2017.7982098

[18] CIGRE TB 496 Brochure CIGR_E 496. Recommendations for Testing DC Extruded Cable Systems for Power Transmission at a Rated Voltage up to $500 \mathrm{kV}$. Prepared by CIGR_E Working Group B132, April 2012.

[19] IEC IEC 62067. "Power Cables with Extruded Insulation and Their Accessories for Rated Voltages above $150 \mathrm{kV}$ (Um 1/4 $170 \mathrm{kV}$ ) up to $500 \mathrm{kV}$ (Um 1/4 $550 \mathrm{kV}$ )—Test Method and Requirements." Second edition, November 2011.

[20] Electra n. 189. "Recommendations for Tests of Power Transmission DC Cables for a Rated Voltage up to 800 kV (ELECTRA 72, 1980-Revision). 2000.

[21]Candela R., Di Stefano A., Fiscelli G., Giaconia G.C., "Portable Partial Discharge Detection Device", Patent EP2297589/ WO2009150627, 11 June 2009.

[22]Candela R., Contin A., "A portable instrument for the location and identification of defects generating PD", In Electrical Insulation Conference (EIC), Annapolis, Maryland, 5-8 June 2011, IEEE, pp. 469-473.

[23]Z. Lei, J. Song, M. Tian, X. Cui, C. Li and M. Wen, "Partial discharges of cavities in ethylene propylene rubber insulation," in IEEE Transactions on Dielectrics and Electrical Insulation, vol. 21, no. 4, pp. 1647-1659, August 2014.

[24]S. Whitehead, Dielectric Breakdown of Solids, Clarendon Press Oxford, 1951.

[25]C. Zhou and G. Chen, "Space charge behaviours in polyethylene under combined AC and DC electric fields," 2014 IEEE Conference on Electrical Insulation and Dielectric Phenomena (CEIDP), Des Moines, IA, 2014, pp. 848-851. doi: 10.1109/CEIDP.2014.6995787

[26]R. Bartnikas, "Partial discharges. Their mechanism, detection and measurement," IEEE Transactions on Dielectrics and Electrical Insulation, vol. 9, no. 5, pp. 763-808, Oct 2002. doi: 10.1109/TDEI.2002.1038663
[27]M. Florkowski, B. Florkowska and P. Zydron, "Partial discharge echo obtained by chopped sequence," in IEEE Transactions on Dielectrics and Electrical Insulation, vol. 23, no. 3, pp. 1294-1302, June 2016. doi: 10.1109/TDEI.2015.005487.

[28] R. Schifani, R. Candela and P. Romano, "On PD mechanisms at high temperature in voids included in an epoxy resin," in IEEE Transactions on Dielectrics and Electrical Insulation, vol. 8, no. 4, pp. 589-597, Aug 2001. doi: 10.1109/94.946711.

[29]P. Romano, R. Candela, A. Imburgia, G. Presti, E. R. Sanseverino and F. Viola, "A new technique for partial discharges measurement under DC periodic stress,"2017 IEEE Conference on Electrical Insulation and Dielectric Phenomenon (CEIDP), Fort Worth, TX, 2017, pp. 303-306.

[30]N. Haque, S. Dalai, B. Chatterjee and S. Chakravorti, "Investigations on charge trapping and de-trapping properties of polymeric insulators through discharge current measurements," in IEEE Transactions on Dielectrics and Electrical Insulation, vol. 24, no. 1, pp. 583-591, Feb. 2017.

[31]R. Candela, G. Mirelli, and R. Schifani, "PD recognition by means of statistical and fractal parameters and a neural network," IEEE Transactions on Dielectrics and electrical Insulation, vol. 7, no. 1, pp. 87-94, 2000.

[32]R. Schifani and R. Candela, "A new algorithm for mixed Weibull analysis of partial discharge amplitude distributions," IEEE transactions on dielectrics and electrical insulation, vol. 6, no. 2, pp. 242-249, 1999.

[33]R. Candela, R. Schifani, B. Cerutti, and F. Guastavino, "Identification and separation of multiple and concurrent partial discharge phenomena," in Electrical Insulation and Dielectric Phenomena, 2000 Annual Report Conference on, 2000, vol. 2, pp. 665-669.

[34]R. Candela and P. Romano, "An improved MSD-based method for PD pattern recognition," in 2007 Annual Report-Conference on Electrical Insulation and Dielectric Phenomena, 2007, pp. 204-207.

[35] A. Krivda, "Automated recognition of partial discharges," in IEEE Transactions on Dielectrics and Electrical Insulation, vol. 2, no. 5, pp. 796-821, Oct 1995. doi: 10.1109/94.469976.

[36] Xiaohua Li, Guangning Wu, Xueqin Zhang and Shanshan Bian, "Partial discharge pulse shape detection and analysis under DC condition in typical defect models," 2007 Annual Report - Conference on Electrical Insulation and Dielectric Phenomena, Vancouver, BC, 2007, pp. 188-191. doi: 10.1109/CEIDP.2007.4451593.

[37] P. Romano, T. Hammarström, T. Bengtsson, A. Imburgia, A. Madonia, F. Viola, S. M. Gubanski, "Partial Discharges at Different Voltage Waveshapes: Comparison between two different acquisition systems" IEEE Transactions on Dielectrics and electrical Insulation, vol. 26, no. 2, pp.584-593, April 2018. doi: 10.1109/TDEI.2018.006782 


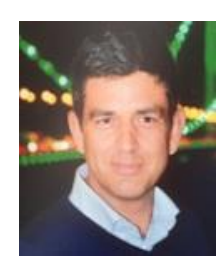

Pietro Romano (M'14-SM'17) received the MSc. and Ph.D. degrees in Electrical Engineering from University of Palermo, Italy, in 1993 and 1998 respectively. From 1998 to 2001 he worked at CRES Centre of Electronic Research in Sicily on partial discharge measurement research. Since 2001 he has been a researcher in the DEIM Department of the University of Palermo, and teaches Basic Electrical Engineering, Electrotechnics and insulating materials. His research activity is mainly in the field of insulating systems diagnosis, partial discharge measurements, HV Systems, multifactor stress effects and electric field simulations, and partially-shaded PV Systems. He is the head of the L.E.PR.E. HV laboratory of Palermo University.

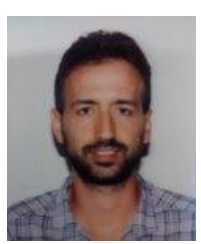

Antonino Imburgia was born in Palermo, Italy, on 28 April 1987. He received the M.Sc. degree in electrical engineering from the University of Palermo in 2014. Since 2015 he has been a Ph.D. student in the DEIM Department of the University of Palermo. His current research is in the field of HVDC, distribution of space charge in solid dielectrics and its behavior, dielectrics and electrical insulating systems diagnosis, space charge and partial discharge measurements.

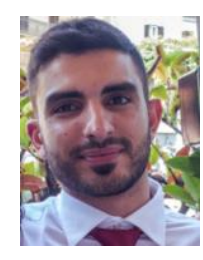

Giuseppe Presti was born in Palermo, Italy, on 14 May 1988. He received the M.Sc. degree in Electrical Engineering from the University of Palermo in 2016. During his academic career he analyzed problems related to insulating materials, and partial discharge physics and measurements. His thesis research was concerned with partial discharge detection in solid dielectrics diagnostic under DC voltage.

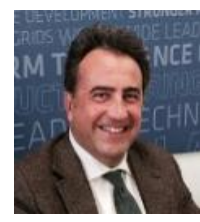

Roberto Candela has conducted research in the dielectric laboratory of the University of Palermo since 1995 , in the partial discharge field, with particular reference to the development of equipment and software aimed at the analysis and measurement of partial discharge in solid insulation. From 2001 he has been a researcher in the DEIM Department of the University of Palermo, and teaches electrotechnics and insulating materials. Since 2012 he has been CEO of Prysmian Electronics, part of Prysmian Group. 